ris und Prof. Dr. Krzystof Pietrzak vom IST Austria für ihr Authentifizierungsprotokoll "LaPIN“. Das nicht auf Blockchiffren basierende Protokoll ist sehr effizient und wesentlich platzsparender als vergleichbar sichere Challenge-Response-Protokolle. Dadurch eignet es sich z. B. ideal für preiswerte RFIDs in der Massenanwendung. Es ist beweisbar sicher und kann nach heutigem Wissensstand selbst von Quantencomputern nicht gebrochen werden. Das Protokoll soll ohne Patent als Standard vorgeschlagen werden.

ForBild: IT-Forensik unterstützt Kampf gegen Kinderpornographie

Ein weiterer zweiter Preis geht an Dr. Huajian Liu, Dr. Martin Steinebach und York Yannikos vom Fraunhofer-Institut für Sichere Informationstechnologie für ihre Entwicklung „ForBild“ zur forensischen Bilderkennung durch robuste Hashs. Die optimierte Technik nutzt nicht die Dateieigenschaften zur Bilderkennung, sondern orientiert sich an der menschlichen Wahrnehmung. Veränderungen der Größe, des Rauschfaktors oder des Dateiformats stören dabei nicht. Das Verfahren wird beispielsweise eingesetzt, um Kriminalbeamten bei der Sichtung von kinderpornographischem Material zeitlich und psychisch zu entlasten.

\section{BSI und if(is) stellen App für IT-Sicherheit zur Verfügung}

Das Bundesamt für Sicherheit in der Informationstechnik (BSI) und das Institut für Internet-Sicherheit der Westfälischen Hochschule in Gelsenkirchen (if(is) stellen die aktualisierte Version der App „securityNews" jetzt mit der mobilen Version der BSI-Schwachstellenampel (https://www.cert-bund.de/schwachstellenampel) zur Verfügung. Die App richtet sich an alle Internetnutzer und bietet neben Sicherheitshinweisen einen Überblick zu aktuellen Gefahren im Netz und wirksamen Gegenmaßnahmen.

Die integrierte Schwachstellenampel des BSI warnt vor Sicherheitslücken in gängiger Standardsoftware, die sowohl bei Privatanwendern und in Unternehmen im Einsatz ist. Anwender und Administratoren können damit erkennen, bei welcher Software noch keine Sicherheits-Updates zur Verfügung stehen. Zu jeder Standardsoftware zeigt die Ampel die Anzahl und Schwere der Sicherheitslücken auf. Darüber hinaus hilft die App mit aktuellen Sicherheitshinweisen der if(is)-Experten zu gängigen Softwareprodukten, das Zeitfenster zwischen der eingetretenen Sicherheitslücke und dem Einspielen der Updates auf ein Minimum zu reduzieren.

Die kostenfreie App „securityNews" steht sowohl für Apple-Geräte wie iPhone und iPad als auch für Android-Smartphones und Tablets zur Verfügung. Zusätzlich kann securityNews auch als EMail-Service oder Web-App auf der Internetseite www.it-sicherheit. de des if(is) kostenfrei bezogen werden.

Ziel des gemeinsamen Projektes, das im Rahmen der Allianz für Cyber-Sicherheit (https://www.allianz-fuer-cybersicherheit.de) stattfindet, ist es, einen einfach nutzbaren mobilen Informationsservice zur Erhöhung der IT-Sicherheit anzubieten. „Mit der Allianz fördern und gestalten wir aktiv die Cyber-Sicherheit in Deutschland. Die App „securityNews" ist ein Beispiel für die erfolgreiche Zusammenarbeit innerhalb der Allianz. Mit der kostenfreien App bieten wir nun allen Internetnutzern einen mobilen Service für mehr Informationssicherheit", so Hartmut Isselhorst, Abteilungsleiter CyberSicherheit im BSI.

\section{certBox.org - Der Zertifikatsserver in der Cloud}

Die Secardeo GmbH hat ihren frei zugänglichen Zertifikatsserver certBox.org weiterentwickelt und über 80 PKI-Directories angeschlossen. Hier sind Zertifikate von mehreren Millionen Benutzern und über 300 Root-CAs zu finden. Damit ist certBox.org die führende Plattform zur Suche und Veröffentlichung von digitalen Zertifikaten.

Dieser Zertifikatsserver löst das grundsätzliche Problem der Verschlüsselung: Die Beschaffung der benötigten Public Keys. Mit certBox.org kann ein Anwender komfortabel nach X.509-Zertifikaten oder PGP Keys suchen und eigene Zertifikate veröffentlichen. Die Suche kann einfach über einen Web-Browser oder automatisch mit einem E-Mailprogramm wie z.B. Outlook durchgeführt werden. Auch die Anbindung an ein gängiges Secure E-Mail Gateway ist problemlos möglich. Die Suchanfragen werden lokal bedient oder gezielt an die ständig wachsende Zahl der angeschlossenen Zertifikatsverzeichnisse weitergeleitet. Mit ausgeklügelten Sicherheitsmechanismen wird der Zugriff auf die Benutzerdaten geschützt.

Mit certBox.org können Anwender sofort und komfortabel verschlüsselte E-Mails an beliebige Partner versenden. Der Nutzen wird durch die globale Erreichbarkeit drastisch erhöht und E-Mailverschlüsselung wird zur Selbstverständlichkeit! Die Plattform certBox.org kann von Einzelanwendern und kleinen Teams kostenlos genutzt werden. Unternehmen können die Möglichkeiten über einen kostenlosen Demozugang erproben.

Die hier eingesetzte Secardeo certBox 2 Appliance ist der führende Zertifikatsserver am Markt und hat sich im Einsatz bei sehr großen PKlen wie Siemens oder der European Bridge CA von TeleTrusT bewährt. Die certBox ist zum Einsatz im Unternehmen als Appliance, Virtual Appliance und als Cloud Service (SaaS) verfügbar.

Weitere Informationen: www.certbox.org .

\section{TeleTrusT - Bundesverband IT-Sicherheit e.V.: Stabile Entwicklung}

Auf seiner Jahresmitgliederversammlung am 30.11.2012 in Berlin zog der Bundesverband IT-Sicherheit (TeleTrusT) eine erfolgreiche Bilanz des zurückliegenden Jahres. Mit einem Zuwachs von 40 neuen Mitgliedern, einem Ausbau der Partnerschaften im In- und Ausland und zahlreichen Aktivitäten befindet sich der Verband weiter auf Expansionskurs, sieht die Grundlage für diese Entwicklung aber auch vor allem in der Aktualität des Themas IT-Sicherheit.

Auf der TeleTrusT-Jahresmitgliederversammlung wurde der amtierende Vorstand einstimmig für eine weitere Amtsperiode wiedergewählt. Alter und neuer Vorsitzender ist Prof. Dr. Norbert Pohlmann (Direktor des Instituts für Internet-Sicherheit an der Westfälischen Hochschule), stellvertretender Vorsitzender Dr. Rainer Baumgart (Vorstandsvorsitzender der secunet Security Networks AG). Weiterhin im Vorstand vertreten und einstimmig bestätigt sind Ammar Alkassar (CEO der Sirrix AG) und Dr. Guido von der Heidt (Siemens AG, Corporate Information Technology). 